\title{
M. S. Sultanakhmedov \\ Cauchy problem for the difference equation and Sobolev orthogonal functions on the finite grid, generated by discrete orthogonal functions
}

We consider the system of functions $\psi_{1, n}(x, N)(n=0,1, \ldots, N)$, orthonormal in Sobolev sense and generated by a given orthonormal on finite grid $\Omega_{N}=\{0,1, \ldots, N-1\}$ system of functions $\psi_{n}(x, N)(n=0,1, \ldots, N-1)$. These new functions are orthonormal with respect to the inner product of the following type: $\langle f, g\rangle=f(0) g(0)+\sum_{j=0}^{N-1} \Delta f(j) \Delta g(j) \rho(j)$. It is shown that the finite Fourier series by the functions $\psi_{1, n}(x)$ and their partial sums are convenient and a very effective tool for the approximate solution of the Cauchy problem for nonlinear difference equations.

Bibliography: 17 items.

Рассматривается система функций $\psi_{1, n}(x, N)(n=0,1, \ldots, N)$, ортонормированная по Соболеву и порожденная заданной ортонормированной на конечной сетке $\Omega_{N}=\{0,1, \ldots, N-1\}$ системой функций $\psi_{n}(x, N)$ $(n=0,1, \ldots, N-1)$. Эта новая система является ортонормированной в смысле скалярного произведения следующего вида: $\langle f, g\rangle=f(0) g(0)+$ $\sum_{j=0}^{N-1} \Delta f(j) \Delta g(j) \rho(j)$. Показано, что конечные ряды Фурье по системе функций $\psi_{1, n}(x, N)$ и их частичные суммы являются удобным и весьма эффективным инструментом приближенного решения задачи Коши для нелинейных разностных уравнений.

Библиография: 17 названий.

Keywords: Sobolev orthogonal functions; functions orthogonal on the finite grid; finite grid; uniform grid; approximation of discrete functions; mixed series by the functions orthogonal on a uniform grid; iterative process for the approximate solution of difference equations.

Ключевые слова: функции, ортогональные по Соболеву; функции, ортогональные на сетке; приближение дискретных функций; смешанные ряды по функциям, ортогональным на равномерной сетке; итерационный процесс для приближенного решения разностных уравнений.

\section{Introduction}

For functions $f$ and $g$, defined on the finite grid $\Omega_{N+r}=\{0,1, \ldots, N+r-1\}$, we introduce the Sobolev-type inner products of the following form

$$
\langle f, g\rangle=\sum_{k=0}^{r-1} \Delta^{k} f(0) \Delta^{k} g(0)+\sum_{j=0}^{N-1} \Delta^{r} f(j) \Delta^{r} g(j) \rho(j),
$$


where $\rho=\rho(j)\left(j \in \Omega_{N}\right)$ - discrete weight function. We assume that $\sum_{k=0}^{r-1} \Delta^{k} f(0) \Delta^{k} g(0)=0$ when $r=0$, while in other cases $(r \geqslant 1)$ this expression helps to «control» the behaviour of the corresponding Sobolev orthogonal functions of the discrete variable at the point $x=0$.

The interest to the systems of functions orthogonal in Sobolev sense is growing steadily (see [1] - [6] and the literature cited there), and this can be explained, among other things, by the fact that this systems proved to be a convenient tool for solving boundary-value problems of differential and difference equations.

In current paper we show that systems of discrete functions orthonormal in the Sobolev sense on finite grid can be used for approximate solution of difference equations (including nonlinear ones). The solution is found in the form of partial sum of the series by these functions.

The central idea of the approach under consideration is to construct an iterative process for the approximate determination of the unknown coefficients of mentioned decomposition of the difference equation solution. An important role in the proof of the constructed iterative process convergence is played by the properties of functions orthonormal on Sobolev and generated by a given system of functions, orthonormal on discrete grid.

\section{Systems of discrete functions orthonormal on Sobolev, generated by orthonormal system of functions}

Here we gather some notation and results, which we will need hereinafter.

Let $\left\{\psi_{k}(x)\right\}_{k=0}^{\infty}$ be a given system of functions orthonormal on the discrete grid $\Omega_{N}=\{0,1, \ldots, N-1\}$ with weight $\rho(x)$. For some real number $a$ and integer $r \geqslant 1$ we assume that $a^{[r]}=a(a-1) \cdots(a-r+1), a^{[0]}=1$, and consider the following functions

$$
\begin{gathered}
\psi_{r, k}(x)=\frac{x^{[k]}}{k !}, k=0,1, \ldots, r-1, \\
\psi_{r, k+r}(x)=\left\{\begin{array}{ll}
\frac{1}{(r-1) !} \sum_{t=0}^{x-r}(x-1-t)^{[r-1]} \psi_{k}(t), & x \in \Omega_{N+r} \backslash \Omega_{r}, \\
0, & x \in \Omega_{r},
\end{array}\right\}, k \in \Omega_{N},
\end{gathered}
$$

which are defined on the grid $\Omega_{N+r}=\{0,1, \ldots, N+r-1\}$. Following [13], we will call the system of function $\psi_{r, n}(x, N)\left(r=1,2, \ldots ; n \in \Omega_{N+r}\right)$ a system, orthonormal on Sobolev with respect to the inner product (1) and generated by system $\left\{\psi_{k}(x)\right\}_{k=0}^{\infty}$.

For function $f=f(x)$ operator of finite difference $\Delta f$ shall be considered as follows: $\Delta f(x)=f(x+1)-f(x)$, and for higher orders $\Delta^{\nu+1} f(x)=\Delta \Delta^{\nu} f(x)$, $\nu \geqslant 1$. In paper [13] some useful difference properties of the functions defined by (2) $-(3)$ were investigated.

Lemma A. The following equalities hold $\left(r \geqslant 1, \nu \geqslant 1, x \in \Omega_{N+r-\nu}\right)$ :

$$
\Delta^{\nu} \psi_{r, k}(x)= \begin{cases}\psi_{r-\nu, k-\nu}(x), & \nu \leqslant \min \{k, r\} \\ \Delta^{\nu-r} \psi_{k-r}(x), & \nu>r, k \geqslant r \\ 0, & \nu, r>k\end{cases}
$$


where $\psi_{0, k}(x)=\psi_{k}(x)$.

For some integer $r \geqslant 0$ we denote by $l_{\rho}^{N+r}$ the space of discrete functions $f=f(x)$, defined on the grid $\Omega_{N+r}$, where the inner product $\langle f, g\rangle$ is introduced by (1). The problem of orthogonality, normality and completeness of system $\left\{\psi_{r, k}(x)\right\}_{k=0}^{N+r-1}$ in space $l_{\rho}^{N+r}$ were investigated in [13].

TheOREM B. Suppose that functions $\psi_{k}(x)(k=0,1, \ldots, N-1)$ form in $l_{\rho}^{N}=$ $l_{\rho}^{N+0}$ a complete orthonormal system with weight $\rho(x)$. Then system $\left\{\psi_{r, k}(x)\right\}_{k=0}^{N+r-1}$, generated by the system $\left\{\psi_{k}(x)\right\}_{k=0}^{N-1}$ with the help of equalities (2) $-(3)$, is complete in $l_{\rho}^{N+r}$ and orthonormal with respect to the inner product (1).

From Theorem B it follows that functions $\psi_{r, k}(t)$ form orthonormal basis in the space $l_{\rho}^{N+r}$, therefore for the arbitrary function $f(x) \in l_{\rho}^{N+r}$ we can write the equality

$$
f(x)=\sum_{k=0}^{\infty}\left\langle f, \psi_{r, k}\right\rangle \psi_{r, k}(x)
$$

which is the finite Fourier series for the function $f(x)$ by the system $\left\{\psi_{r, k}(t)\right\}_{k=0}^{N+r-1}$, orthonormal on Sobolev. Since the Fourier coefficients $\left\langle f, \psi_{r, k}\right\rangle$ are of the form

$$
\begin{gathered}
f_{r, k}=\left\langle f, \psi_{r, k}\right\rangle=\sum_{\nu=0}^{r-1} \Delta^{\nu} f(0) \Delta^{\nu} \psi_{r, k}(0)=\Delta^{k} f(0), \quad k=0, \ldots, r-1 ; \\
f_{r, k}=\left\langle f, \psi_{r, k}\right\rangle=\sum_{j=0}^{N-1} \Delta^{r} f(j) \Delta^{r} \psi_{r, k}(j) \rho(j)= \\
\sum_{j=0}^{N-1} \Delta^{r} f(j) \psi_{k-r}(j) \rho(j), \quad k=r, \ldots, N+r-1 ;
\end{gathered}
$$

then we can write the (5) in the following mixed form

$$
f(x)=\sum_{k=0}^{r-1} \Delta^{k} f(0) \frac{x^{[k]}}{k !}+\sum_{k=r}^{N+r-1} f_{r, k} \psi_{r, k}(x), x \in \Omega_{N+r} .
$$

Therefore Fourier series by the system $\left\{\psi_{r, k}(t)\right\}_{k=0}^{\infty}$ following [7] - [12] we will call mixed series by the original orthonormal system $\left\{\psi_{k}(t)\right\}_{k=0}^{\infty}$. Here we note some important properties of mixed series (6) and their partial sums of the following form

$$
\mathcal{Y}_{r, n}(f, x)=\sum_{k=0}^{r-1} \Delta^{k} f(0) \frac{x^{[k]}}{k !}+\sum_{k=r}^{n} f_{r, k} \psi_{r, k}(x) .
$$

Taking into account the equalities (4), from (6) - (7) for $0 \leqslant \nu \leqslant r-1$ and $x \in \Omega_{N+r-\nu}$ we can write out:

$$
\Delta^{\nu} f(x)=\sum_{k=0}^{r-\nu-1} \Delta^{k+\nu} f(0) \frac{x^{[k]}}{k !}+\sum_{k=r-\nu}^{N+r-\nu-1} f_{r, k+\nu} \psi_{r-\nu, k}(x),
$$




$$
\begin{gathered}
\Delta^{\nu} \mathcal{Y}_{r, n}(f, x)=\sum_{k=0}^{r-\nu-1} \Delta^{k+\nu} f(0) \frac{x^{[k]}}{k !}+\sum_{k=r-\nu}^{n-\nu} f_{r, k+\nu} \psi_{r-\nu, k}(x), \\
\Delta^{\nu} \mathcal{Y}_{r, n}(f, x)=\mathcal{Y}_{r-\nu, n-\nu}\left(\Delta^{\nu} f, x\right), \\
\Delta^{\nu} \mathcal{Y}_{r, n}(f, 0)=\Delta^{\nu} f(0), \quad 0 \leqslant \nu \leqslant r-1 .
\end{gathered}
$$

Moreover, from (4) and (6) we have

$$
\Delta^{r} f(x)=\sum_{k=0}^{N-1} f_{r, r+k} \psi_{k}(x), \quad x \in \Omega_{N} .
$$

\section{On the representation of the difference equation solution as Fourier series by functions $\psi_{1, n}(x)$}

Now we shall consider the problem of finding an approximate solution of difference equation

$$
\Delta y(x)=h f(x, y), \quad y(0)=y_{0}, \quad h>0, \quad x \in \Omega_{N},
$$

in the form of Fourier sum by the system $\left\{\psi_{1, n}(x)\right\}_{n=0}^{N}$, orthonormal on Sobolev and generated from orthonormal system of functions $\left\{\psi_{n}(x)\right\}_{n=0}^{N-1}$ with the help of equalities $(2)-(3)$. We notice, that similar problem in case when function $f(x, y(x))$ is given on infinite grid $\Omega=\{0,1,2, \ldots\}$ instead of $\Omega_{N}$ was considered in paper [17]. We also should mention that the results obtained in Theorem 1 can be generalized to the case of systems of difference equations of the form

$$
\left\{\begin{array} { l } 
{ \Delta y _ { 1 } ( x ) = h f _ { 1 } ( x , y _ { 1 } , y _ { 2 } , \ldots , y _ { m } ) , } \\
{ \Delta y _ { 2 } ( x ) = h f _ { 2 } ( x , y _ { 1 } , y _ { 2 } , \ldots , y _ { m } ) , } \\
{ \cdots } \\
{ \Delta y _ { m } ( x ) = h f _ { m } ( x , y _ { 1 } , y _ { 2 } , \ldots , y _ { m } ) , }
\end{array} \quad \left\{\begin{array}{ll}
y_{1}(0)=y_{0}^{1}, & \\
y_{2}(0)=y_{0}^{2}, & \\
\ldots & \\
y_{m}(0)=y_{0}^{m}, &
\end{array}\right.\right.
$$

But for the purpose of easier recording we confine ourselves to consideration of the Cauchy problem (8), where the function $f(x, y)$ is assumed to be given on the Cartesian product $\Omega_{N} \times \mathbb{R}$. It is required to approximate with a given accuracy the function $y=y(x)$, which is the solution of the problem (8).

We suppose that system $\left\{\psi_{n}(x)\right\}_{n=0}^{N-1}$ satisfies the conditions of Theorem B. Since $f(x, y)$ is defined on the set $\Omega_{N} \times \mathbb{R}$, then $\Delta y(x)$ is defined on $\Omega_{N}$, and, consequently, $y(x) \in l_{\rho}^{N+1}$. Therefore, we can represent $y(x)$ in the form of a finite series

$$
y(x)=y(0)+\sum_{j=0}^{N-1} y_{1, j+1} \psi_{1, j+1}(x), \quad\left(x \in \Omega_{N+1}\right),
$$

where coefficients

$$
y_{1, j+1}=\sum_{t=0}^{N-1} \Delta y(t) \psi_{k}(t) \rho(t), \quad(j=0,1, \ldots, N-1),
$$


can also be regarded as coefficients of the function's $g(t)=\Delta y(t)$ Fourier transform by the original system $\left\{\psi_{k}(x)\right\}_{k=0}^{N-1}$. Thereby, we can write

$$
\Delta y(x)=\sum_{k=0}^{N-1} y_{1, k+1} \psi_{k}(x), \quad x \in \Omega_{N} .
$$

This equality together with (8) gives us

$$
q(x)=f(x, y(x))=\frac{1}{h} \Delta y(x)=\sum_{k=0}^{N-1} c_{k}(q) \psi_{k}(x), \quad x \in \Omega_{N},
$$

where

$$
c_{k}(q)=\frac{1}{h} y_{1, k+1}=\sum_{t=0}^{N-1} f(t, y(t)) \psi_{k}(t) \rho(t), \quad(k=0,1, \ldots, N-1) .
$$

Using this new coefficients, we can rewrite (9) as follows

$$
y(x)=y(0)+h \sum_{j=0}^{N-1} c_{j}(q) \psi_{1, j+1}(x), \quad\left(x \in \Omega_{N+1}\right) .
$$

This expression for $y(x)$ in its turn can be used to rewrite (11) as

$$
c_{k}(q)=\sum_{t=0}^{N-1} f\left[t, y(0)+h \sum_{j=0}^{N-1} c_{j}(q) \psi_{1, j+1}(t)\right] \psi_{k}(t) \rho(t), \quad(k=0,1, \ldots, N-1) .
$$

\section{Construction of iterative process}

To find approximate values of these coefficients $c_{k}(q)$ we will try to construct convergent iterative process. In the space $\mathbb{R}^{N}$ consider the operator $A_{N}^{q}$, which for any vector $C=\left(c_{0}, c_{1}, \ldots, c_{N-1}\right)$ matches a vector $C^{\prime}=\left(c_{0}^{\prime}, c_{1}^{\prime}, \ldots, c_{N-1}^{\prime}\right)$ by the rule

$$
c_{k}^{\prime}=\sum_{t=0}^{\infty} f\left[t, y(0)+h \sum_{j=0}^{N-1} c_{j} \psi_{1, j+1}(t)\right] \psi_{k}(t) \rho(t), \quad(k=0,1, \ldots, N-1) .
$$

From (13) it follows that vector $C(q)=\left(c_{0}(q), c_{1}(q), \ldots, c_{N-1}(q)\right)$ is a fixed point of the operator $A_{N}^{q}: \mathbb{R}^{N} \rightarrow \mathbb{R}^{N}$. In order to find this vector using the method of simple iterations, it suffices to show that $A_{N}^{q}$ is a contraction mapping in the metric of $\mathbb{R}^{N}$. For this purpose let us consider two vectors $P=\left(p_{0}, p_{1}, \ldots, p_{N-1}\right)$, $Q=\left(q_{0}, q_{1}, \ldots, q_{N-1}\right)$ and their images $P^{\prime}=A_{N}^{q}(P), Q^{\prime}=A_{N}^{q}(Q)$. We have

$$
p_{k}^{\prime}-q_{k}^{\prime}=\sum_{t=0}^{N-1} F_{P, Q}(t) \psi_{k}(t) \rho(t) d t, \quad(k=0,1, \ldots, N-1)
$$


where

$$
F_{P, Q}(t)=f\left[t, y(0)+h \sum_{j=0}^{N-1} p_{j} \psi_{1, j+1}(t)\right]-f\left[t, y(0)+h \sum_{j=0}^{N-1} q_{j} \psi_{1, j+1}(t)\right]
$$

Using Bessel's inequality, from (15) we find

$$
\sum_{k=0}^{N-1}\left(p_{k}^{\prime}-q_{k}^{\prime}\right)^{2} \leqslant \sum_{t=0}^{N-1}\left(F_{P, Q}(t)\right)^{2} \rho(t)
$$

Suppose that for $f(x, y)$ the Lipschitz condition by the variable $y$ holds:

$$
\left|f\left(x, y^{\prime}\right)-f\left(x, y^{\prime \prime}\right)\right| \leqslant \lambda\left|y^{\prime}-y^{\prime \prime}\right|, \quad x \in \Omega_{N},
$$

then from (16) and (18) we get

$$
\left(F_{P, Q}(t)\right)^{2} \leqslant(\lambda h)^{2}\left(\sum_{j=0}^{N-1}\left(p_{j}-q_{j}\right) \psi_{1, j+1}(t)\right)^{2}
$$

and, using the Cauchy - Bunyakovsky inequality, finally we have

$$
\left(F_{P, Q}(t)\right)^{2} \leqslant(\lambda h)^{2} \sum_{k=0}^{N-1}\left(p_{k}-q_{k}\right)^{2} \sum_{j=0}^{N-1}\left(\psi_{1, j+1}(t)\right)^{2} .
$$

Gathering (19) together with (17) gives us

$$
\sum_{k=1}^{N-1}\left(p_{k}^{\prime}-q_{k}^{\prime}\right)^{2} \leqslant(\lambda h)^{2} \sum_{k=0}^{N-1}\left(p_{k}-q_{k}\right)^{2} \sum_{t=0}^{N-1} \sum_{j=0}^{N-1}\left(\psi_{1, j+1}(t)\right)^{2} \rho(t),
$$

or rewritten

$$
\left(\sum_{k=0}^{N-1}\left(p_{k}^{\prime}-q_{k}^{\prime}\right)^{2}\right)^{\frac{1}{2}} \leqslant \kappa_{N}^{\psi} \lambda h\left(\sum_{k=0}^{N-1}\left(p_{k}-q_{k}\right)^{2}\right)^{\frac{1}{2}}
$$

where

$$
\kappa_{N}^{\psi}=\left(\sum_{t=0}^{N-1} \sum_{k=1}^{N-1}\left(\psi_{1, k}(t)\right)^{2} \rho(t)\right)^{\frac{1}{2}} .
$$

The inequality (20) shows that if $\kappa_{N}^{\psi} \lambda h<1$, then $A_{N}^{q}: \mathbb{R}^{N} \rightarrow \mathbb{R}^{N}$ is the contraction mapping, and as a consequence the iterative process $C^{\nu+1}=A_{N}^{q}\left(C^{\nu}\right)$ converges to the point $C(q)$ as $\nu \rightarrow \infty$.

However, in applications it is often more convenient to use as an solution approximation not the whole finite Fourier series (12), but its partial sum of some order $n$ :

$$
y(x) \approx y(0)+h \sum_{j=0}^{n-1} c_{j}(q) \psi_{1, j+1}(x), \quad\left(x \in \Omega_{N+1}\right) .
$$


To find approximate values of first $n$ coefficients of Fourier series decomposition, we will construct new operator $A_{n, N}^{q}: \mathbb{R}^{n} \rightarrow \mathbb{R}^{n}$, which for any vector $\bar{C}=$ $\left(\bar{c}_{0}, \bar{c}_{1}, \ldots, \bar{c}_{n-1}\right)$ matches a vector $\bar{C}^{\prime}=\left(\bar{c}_{0}^{\prime}, \bar{c}_{1}^{\prime}, \ldots, \bar{c}_{n-1}^{\prime}\right)$ by the rule

$$
\bar{c}_{k}^{\prime}=\sum_{t=0}^{N-1} f\left[t, y(0)+h \sum_{j=0}^{n-1} \bar{c}_{j} \psi_{1, j+1}(t)\right] \psi_{k}(t) \rho(t), \quad(k=0,1, \ldots, n-1) .
$$

Then for two vectors $\bar{P}_{n}=\left(\bar{p}_{0}, \bar{p}_{1}, \ldots, \bar{p}_{n-1}\right), \bar{Q}_{n}=\left(\bar{q}_{0}, \bar{q}_{1}, \ldots, \bar{q}_{n-1}\right)$ we can assume $\bar{P}_{n}^{\prime}=A_{n, N}^{q}\left(\bar{P}_{n}\right), \bar{Q}_{n}^{\prime}=A_{n, N}^{q}\left(\bar{Q}_{n}\right)$ and repeating verbatim the reasoning that led us to the inequality (20), we get

$$
\left(\sum_{k=0}^{n-1}\left(\bar{p}_{k}^{\prime}-\bar{q}_{k}^{\prime}\right)^{2}\right)^{\frac{1}{2}} \leqslant \kappa_{N}^{\psi} \lambda h\left(\sum_{k=0}^{n-1}\left(\bar{p}_{k}-\bar{q}_{k}\right)^{2}\right)^{\frac{1}{2}}
$$

Now we can see that under the same condition $\kappa_{N}^{\psi} \lambda h<1$ this new operator $A_{n, N}^{q}$ becomes contraction, and as the consequence the iterative process $C_{n, N}^{\nu+1}=$ $A_{n, N}^{q}\left(C_{n, N}^{\nu}\right)$ converges to some fixed point, which we denote by $\bar{C}_{n, N}(q)=\left(\bar{c}_{0}(q), \ldots, \bar{c}_{n-1}(q)\right)$.

On the other hand we have vector $C_{n, N}(q)=\left(c_{0}(q), \ldots, c_{n-1}(q)\right)$, composed of first $n$ desired Fourier coefficients of the function $q(x)$ by the system $\left\{\psi_{k}\right\}_{k=0}^{N-1}$. Naturally we want to estimate the error that arises when $C_{n, N}(q)$ is replaced by $\bar{C}_{n, N}(q)$. More precisely, it is required to estimate the value

$$
\left\|C_{n, N}(q)-\bar{C}_{n, N}(q)\right\|_{n}=\left(\sum_{j=0}^{n-1}\left(c_{j}(q)-\bar{c}_{j}(q)\right)^{2}\right)^{\frac{1}{2}} .
$$

For this purpose we consider new vector

$$
\tilde{C}_{n, N}(q)=A_{n, N}^{q}\left(C_{n, N}(q)\right)=\left(\tilde{c}_{0}(q), \ldots, \tilde{c}_{n-1}(q)\right),
$$

and by triangle inequality get

$$
\left\|C_{n, N}(q)-\bar{C}_{n, N}(q)\right\|_{n} \leqslant\left\|C_{n, N}(q)-\tilde{C}_{n, N}(q)\right\|_{n}+\left\|\tilde{C}_{n, N}(q)-\bar{C}_{n, N}(q)\right\|_{n} .
$$

Using (23), we can write

$$
\begin{gathered}
\left\|\tilde{C}_{n, N}(q)-\bar{C}_{n, N}(q)\right\|_{n}=\left\|A_{n, N}^{q}\left(C_{n, N}(q)\right)-A_{n, N}^{q}\left(\bar{C}_{n, N}(q)\right)\right\|_{n} \leqslant \\
\kappa_{N}^{\psi} \lambda h\left\|C_{n, N}(q)-\bar{C}_{n, N}(q)\right\|_{n} .
\end{gathered}
$$

From (24) and (25) we deduce

$$
\left\|C_{n, N}(q)-\bar{C}_{n, N}(q)\right\|_{n} \leqslant \frac{1}{1-\kappa_{N}^{\psi} \lambda h}\left\|C_{n, N}(q)-\tilde{C}_{n, N}(q)\right\|_{n} .
$$

To estimate the norm on the right-hand side, we use the Bessel inequality:

$$
\left\|C_{n, N}(q)-\tilde{C}_{n, N}(q)\right\|_{n}^{2} \leqslant \sum_{t=0}^{N-1}\left(F_{C_{N}(q), C_{n, N}(q)}(t)\right)^{2} \rho(t),
$$


where

$$
\begin{gathered}
F_{C_{N}(q), C_{n, N}(q)}(t)= \\
f\left[t, y(0)+h \sum_{j=0}^{N-1} c_{j}(q) \psi_{1, j+1}(t)\right]-f\left[t, y(0)+h \sum_{j=0}^{n-1} c_{j}(q) \psi_{1, j+1}(t)\right] .
\end{gathered}
$$

By the Lipschitz condition (18) we have

$$
\left(F_{C_{N}(q), C_{n, N}(q)}(t)\right)^{2} \leqslant \lambda^{2}\left(\sum_{j=n}^{N-1} h c_{j}(q) \psi_{1, j+1}(t)\right)^{2},
$$

and with the notation (11) we get

$$
\left(F_{C_{N}(q), C_{n, N}(q)}(t)\right)^{2} \leqslant \lambda^{2}\left(\sum_{j=n}^{N-1} y_{1, j+1} \psi_{1, j+1}(t)\right)^{2} .
$$

This estimate together with (27) gives us

$$
\left\|C_{n, N}(q)-\tilde{C}_{n, N}(q)\right\|_{n}^{2} \leqslant \lambda^{2} \sum_{t=0}^{N-1}\left(\sum_{j=n}^{N-1} y_{1, j+1} \psi_{1, j+1}(t)\right)^{2} \rho(t) .
$$

Summing up all the above, we can derive the following result from (26) and (28).

THEOREM 1. For given Cauchy problem (8) suppose function $f(x, y)$ is defined on the Cartesian product $\Omega_{N} \times \mathbb{R}$ and satisfies the Lipschitz condition (18); constants $h$ and $\lambda$ satisfy the condition $h \lambda \kappa_{N}^{\psi}<1$, where $\kappa_{N}^{\psi}$ is defined by the equality (21). Introducing notation $q(x)=f(x, y(x))$, we consider $A_{N}^{q}: \mathbb{R}^{N} \rightarrow \mathbb{R}^{N}$ to be the operator, which for any vector $C \in \mathbb{R}^{N}$ matches vector $C^{\prime} \in \mathbb{R}^{N}$ by the rule (14); and $A_{n, N}^{q}: \mathbb{R}^{n} \rightarrow \mathbb{R}^{n}$ is the similar operator, which uses matching rule (22).

Then both the operators $A_{N}^{q}$ and $A_{n, N}^{q}$ are contraction mappings, and consequently there are fixed points $C_{N}(q)=A_{N}^{q}\left(C_{N}(q)\right) \in \mathbb{R}^{N}$ and $\bar{C}_{n, N}(q)=A_{n, N}^{q}\left(\bar{C}_{n, N}(q)\right) \in$ $\mathbb{R}^{n}$. Moreover, if $C_{n, N}(q)$ is a sequence consisting of the first $n$ components of vector $C_{N}(q)$, then following inequality holds

$$
\left\|C_{n, N}(q)-\bar{C}_{n, N}(q)\right\|_{N} \leqslant \frac{\lambda \sigma_{n, N}^{\psi}(y)}{1-h \kappa_{N}^{\psi} \lambda}
$$

where

$$
\sigma_{n, N}^{\psi}(y)=\left(\sum_{t=0}^{N-1}\left(\sum_{j=n+1}^{N} y_{1, j} \psi_{1, j}(t)\right)^{2} \rho(t)\right)^{\frac{1}{2}},
$$

$\left\{y_{1, j}\right\}_{j=1}^{N}-$ Fourier coefficients given by $(10)$.

\section{References}

[1] Iserles A., Koch P.E., Norsett S.P., Sanz-Serna J.M. On polynomials orthogonal with respect to certain Sobolev inner products // J. Approx. Theory. 1991. Vol. 65. Pp. $151-175$. 
[2] Marcellan F., Alfaro M., Rezola M.L. Orthogonal polynomials on Sobolev spaces: old and new directions // Journal of Computational and Applied Mathematics. 1993. Vol. 48. Pp. 113-131.

[3] Meijer H.G. Laguerre polynimials generalized to a certain discrete Sobolev inner product space // J. Approx. Theory. 1993. Vol. 73. Pp. 1-16.

[4] Kwon K.H., Littlejohn L.L. The orthogonality of the Laguerre polynomials $\left\{L_{n}^{(-k)}(x)\right\}$ for positive integers $k / /$ Ann. Numer. Anal. 1995. Issue 2. Pp. 289-303.

[5] Kwon K.H., Littlejohn L.L. Sobolev orthogonal polynomials and second-order differential equations // Rocky Mountain J. Math. 1998. Vol. 28. Pp. 547-594.

[6] Marcellan F., Yuan Xu. On Sobolev orthogonal polynomials // arXiv: 6249v1 [math.C.A] 25 Mar 2014. Pp. 1-40.

[7] Sharapudinov I.I. Approximation of discrete functions and Chebyshev polynomials orthogonal on the uniform grid // Math. Notes. 2000. Vol. 67. Issue 3. Pp. 389-397.

[8] Sharapudinov I.I. Mixed series in ultraspherical polynomials and their approximation properties // Sbornik: Mathematics. 2003. Vol. 194. Issue 3. Pp. 423-456.

[9] Sharapudinov I.I. Smeshannie ryadi po ortogonalnim poliminam. Izdatelstvo Dagestanskogo nauchnogo centra. Makhachakala. 2004. Pp. 1-176. (in Russian)

[10] Sharapudinov I.I. Mixed series of Chebyshev polynomials orthogonal on a uniform grid // Math. Notes. 2005. Vol. 78. Issue 3-4. Pp. 403-423.

[11] Sharapudinov I.I. Approximation properties of mixed series in terms of Legendre polynomials on the classes $W^{r} / /$ Sbornik: Mathematics. 2006. Vol. 197. Issue 3. Pp. 433-452.

[12] Sharapudinov I.I., Sharapudinov T.I. Mixed series of Jacobi and Chebyshev polynomials and their discretization // Math. Notes. 2010. Vol. 88. Issue 1. Pp. 112-139.

[13] Sharapudinov I.I., Sharapudinov T.I. Polynomials orthogonal in the Sobolev sense, generated by Chebyshev polynomials orthogonal on a mesh // Russian Mathematics. 2017. Vol. 61, Iss. 8. Pp. 59-70.

[14] Sharapudinov I.I. O priblizhenii resheniya zadachi Koshi dlya nelineynih sistem ODU posredstvom ryadov Furye po funkciyam, ortogonal'nym po Sobolevu // Daghestan Electronic Mathematical Reports. 2017. Vol. 7. Pp. 66-76. (in Russian)

[15] Sharapudinov I.I., Magomedov S.R. Systems of functions orthogonal in the sense of Sobolev associated with Haar functions and the Cauchy problem for ODEs // Daghestan Electronic Mathematical Reports, 2017. Vol. 7. Pp. 1-15.

[16] Sharapudinov I.I., Gadzhieva Z.D., Gadzhimirzaev R.M. Difference equations and Sobolev orthogonal polynomials, generated by Meixner polynomials // Vladikavkaz Mathematical Journal. 2017. Vol. 19. Issue 2. Pp. 58-72. (in Russian)

[17] Sharapudinov I.I., Gadzhieva Z.D., Gadzhimirzaev R.M. Sobolev orthogonal functions on the grid, generated by discrete orthogonal functions and the Cauchy problem for the difference equation // Daghestan Electronic Mathematical Reports. 2017. Vol. 7. Pp. 29-39. 